\title{
Distribution of current density across the active area at various stoichiometry ratios using the JRC ZEROVCELL single cell PEM fuel cell testing hardware
}

\author{
Tomasz Bednarek* \\ European Commission, Joint Research Centre (JRC), Directorate C Energy Transport and Climate, The Netherlands
}

\begin{abstract}
The performance of the PEM fuel cell directly depends on the partial pressure of provided reactants, namely hydrogen and oxygen. Since reactants are consumed in the fuel cell reaction, partial pressure of reactants decreases in the direction of reactants flow. This well-known mechanism makes the performance of the fuel cell dependent on the stoichiometry ratios of input reactants. The JRC ZEROVCELL, a single cell PEM fuel cell testing setup, is developed to provide as much as possible uniform operating conditions at the $10 \mathrm{~cm}^{2}$ active area specimen, hence giving uniform current density across the active area of the cell. To investigate what is the real gradient of current density across the active area for the JRC ZEROVCELL at various reactant stoichiometry ratios, segmented bi-polar plates and current collectors are developed.

This study presents experimental investigation of the current density distribution across the active area of the JRC ZEROVCELL setup at range of reactant stoichiometry ratios from $\lambda=2$ up to $\lambda=15$. Current density gradients are considered along the gas flow as well as in the transverse direction. The experimental results show that the current density gradient across the active area, although dependant on the reactants stoichiometry ratios, is relatively small as compared with a wide range of investigated stoichiometry ratios.
\end{abstract}

\section{Introduction}

The JRC ZEROVCELL has been developed [1,2] with the purpose to minimise the influence of the testing hardware on the performance and durability test results. The features of this hardware setup ensure that the tested Membrane Electrode Assembly (MEA) is exposed to possibly uniform distribution of operating conditions in terms of temperature, pressure and reactant gases composition across the active area, therefore showing the "real" characteristics of the tested specimen. Hence, the JRC ZEROVCELL has the potential to be used as a reference testing hardware for PEM single cell testing.

However, the presented up-to-date results $[1,3]$, do not provide experimentally measured voltage and current density distribution across the active area of the MEA.

The aim of the current study is to provide an information how reactant stoichiometry ratios influence current density distribution across the active area of the MEA. For this purpose, a segmented current collector has been developed, allowing to measure voltage and current distribution along as well across the parallel channels at the MEA active area.

\section{Design criteria and hardware setup}

Measurements of voltage and current distribution across the active area of the tested MEA required a modification of the current collector and the bi-polar plate of the JRC ZEROVCELL testing setup. The other parts of the testing hardware remained unchanged. The experiments were done with regular non-segmented MEA.

The principle of the segmented measuring circuits are presented in Fig. 1. It is assumed the experiment is carried out using a single electronic bank, hence all gradients of current and voltage result from the features and operation of the testing setup. It can be seen in Fig. 1, that voltage and current measurement circuits are separated. This is to avoid possible effects of the current measurement circuit to the voltage measurements. The current measurements are done using high-precision shunt resistors with additional possibility of calibration.

The sketch of measuring segments is shown in Fig.2a. The current collector, presented in Fig. 2b, is a Printed Circuit Board (PCB) with imprinted voltage sensing and current lines and sockets for sensing and current cables. The voltage sensing lines and current lines are as much as

\footnotetext{
* Corresponding author: Tomasz.Bednarek@ec.europa.eu
} 


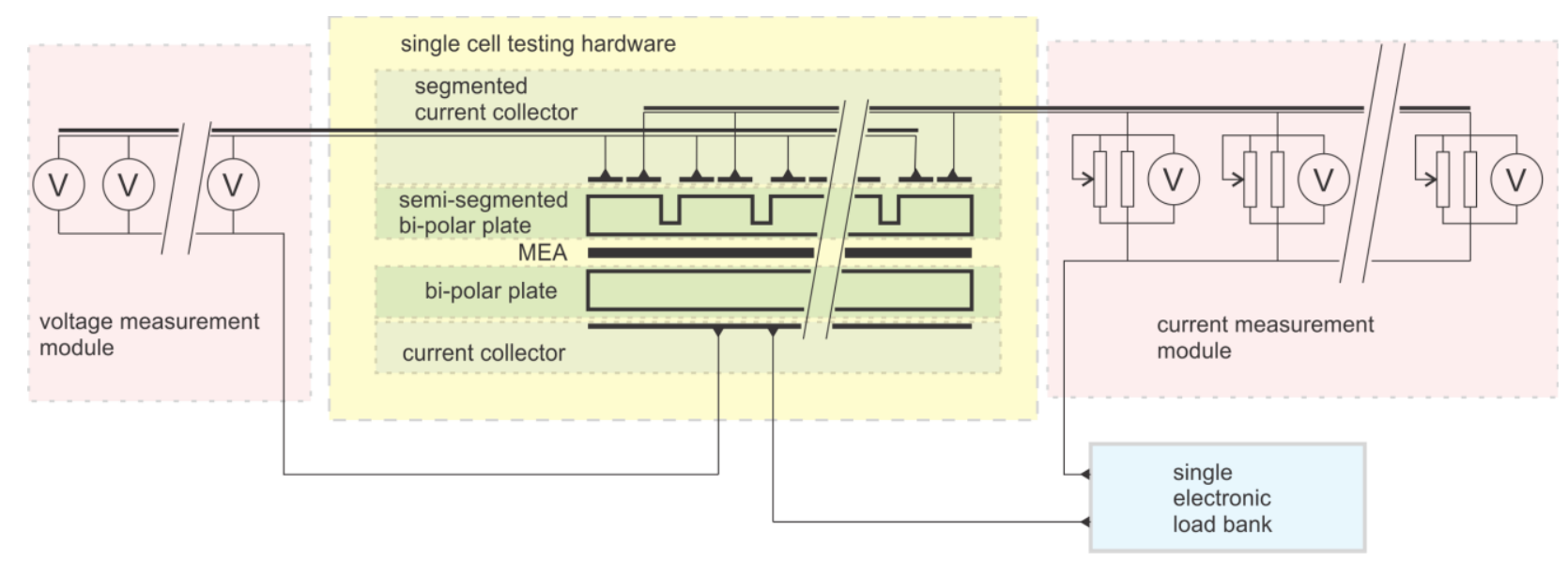

Fig. 1. Circuits for current and voltage distributions measurements.

possible separated to minimise all possible interferences, i.e. for Electro-Impedance Spectroscopy (EIS) measurements. In this purpose, the voltage senses socket and current lines socket are placed on separate sides of the testing setup.

Current measurement shunts are located in separated current measurement module. Such configuration avoids effects of shunts on voltage measurements and allows for more compact design of the current collector PCB. Additional calibration of each individual shunt is possible using multi-turn calibration potentiometers.

Both, voltage and current recordings are done using external data acquisition system NI CompactDAQ. It is worth to mention, that relatively high currents produced by the JRC ZEROVCELL, in extreme cases up to 50A, turn out in considerable electromagnetic fields around the testing setup. Such conditions make precise measurements difficult. The correct shielding and earth grounding of all current and sensing lines was of

a)

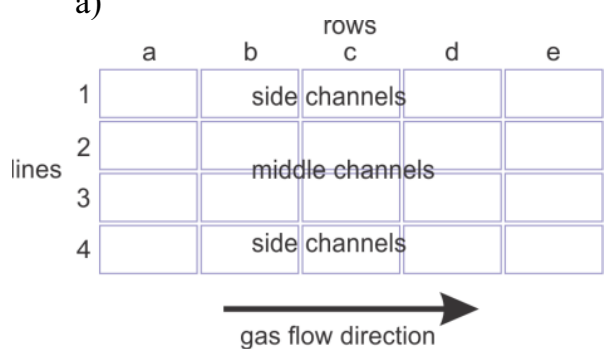

b)

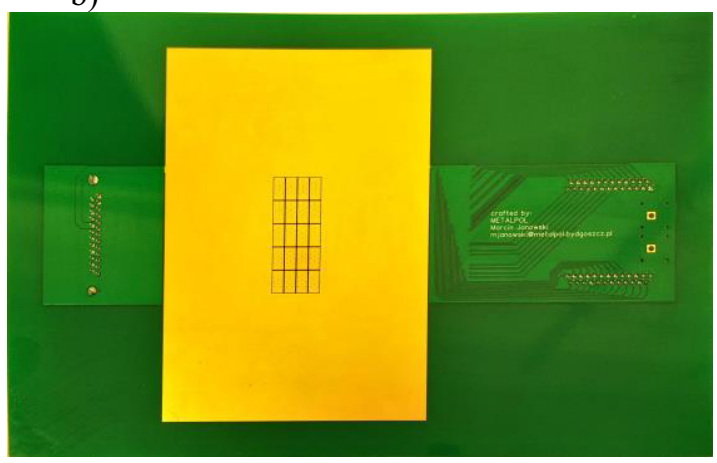

Fig. 2. Segmented current collectors, (a) localisation of segments, (b) current collector PCB plate. importance there. Finally, it was possible to make voltage measurements with accuracy $\pm 1 \mathrm{mV}$ and current measurements $\pm 3 \mathrm{~mA}$. Additional post-process time averaging of the obtained results allowed further improvement of the obtained results accuracy.

As the first try, due to difficulties to achieve full segmentation, a semi-segmentation of the bi-polar plate was done, see Fig. 3. In the solid block of graphite bipolar plate, on the opposite side to the gas channel grooves, segments were milled matching to the measuring points in the segmented current collector. Such approach, considering also non-segmented MEA used for tests, provides uniform voltage across the whole active area. Therefore, segmented measurements are limited only to current distribution.

It is worth to be noted, that such semi-segmented bipolar plate approach with uniform voltage distribution is suitable for both, potentiostatic as well as galvanostatic operation modes. In galvanostatic mode, although voltage across the active area is uniform, the result consist of current distribution across the active area, while integral of current going through each segment gives the total current applied to the cell.

\section{Results and discussion}

The JRC ZEROVCELL testing setup with segmented current collector installed in the testing bench is presented in Fig. 4. The experiments were done using the $10 \mathrm{~cm}^{2}$ (20 mm width, $50 \mathrm{~mm}$ length) active area MEA. The active area was divided into 20 equal segments, 0.5 $\mathrm{cm}^{2}(5 \times 10 \mathrm{~mm})$ each. There are 4 segments in the transverse direction to the gas channels and 5 segments along gas channels, see segmentation sketch in Fig. 2. Operating conditions for the tests are listed in Fig. 5.

In order to get reference performance of the tested MEA, polarisation IV curves experiments were made for range reactant stoichiometry ratios, from $\lambda_{a n}=4, \lambda_{\text {cath }}=5$ up to $\lambda_{a n}=12, \lambda_{\text {cath }}=15$. The polarisation curves were obtained at galvanostatic mode.

It can be seen, Fig. 5, that the effects of stoichiometry ratio on the performance of the cell are visible at rather low current densities, up to $1.0 \mathrm{Acm}^{-2}$ and at the highest current densities above $2.5 \mathrm{Acm}^{-2}$. At the moderate 

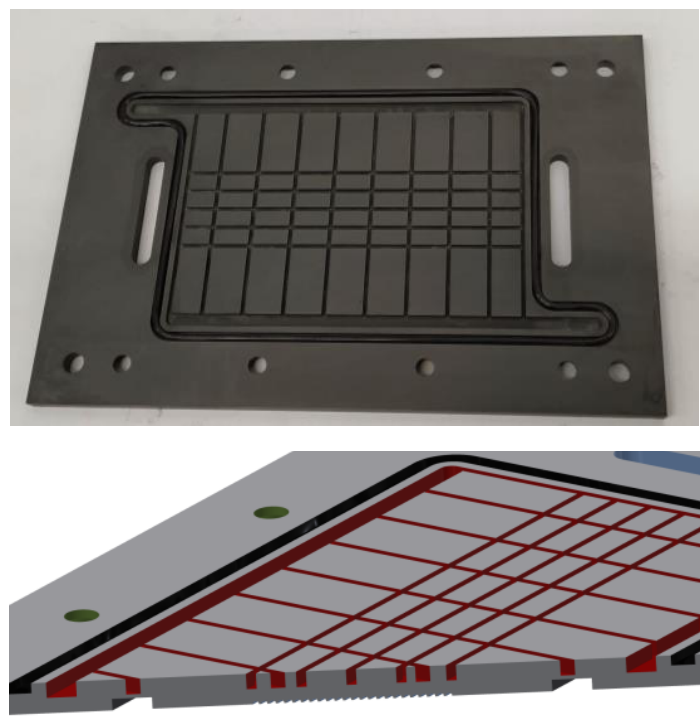

Fig. 3. Semi-segmented graphite bi-polar plate.

current densities between $1.0 \mathrm{Acm}^{-2}$ and $2.5 \mathrm{Acm}^{-2}$, the effects of the reactant stoichiometry on the performance of the MEA are less pronounced.

At the lowest current densities, namely $0.5 \mathrm{Acm}^{-2}$ and $1.0 \mathrm{Acm}^{-2}$, the difference between the best performance $\left(\lambda_{a n}=12, \lambda_{\text {cath }}=15\right)$ and the lowest $\left(\lambda_{a n}=4, \lambda_{\text {cath }}=5\right)$ is about $14 \mathrm{mV}$ and $17 \mathrm{mV}$ respectively. Although those differences are clearly visible on the plot, Fig. 5, the variations of the total output power density do not exceed $\pm 1.5 \%$ and $\pm 1.3 \%$ at current densities $0.5 \mathrm{Acm}^{-2}$ and 1.0 $\mathrm{Acm}^{-2}$ respectively.

Considering that at moderate current densities, from $1.5 \mathrm{Acm}^{-2}$ up to $2.5 \mathrm{Acm}^{-2}$, the effects of reactants stoichiometry ratios on MEA performance nearly disappear (variations below $0.6 \%$ ), the effects of reactant stoichiometry ratios at lower current densities might be associated with low mass flow rate of reactant gasses. Due to employed parallel channels flow field pattern, at low current densities, the pressure drop or reactant gasses is relatively small, which in turn limits a driving force for liquid water removal. Therefore, the product liquid water at the active area of the MEA is evacuated less effectively as compared with higher mass flow rates at moderate current densities.

At high current densities above $3.0 \mathrm{Acm}^{-2}$, it is observed that reactant stoichiometry ratios start affecting the cell performance. At the highest current density, namely $4.0 \mathrm{Acm}^{-2}$, the difference between the highest performance at $\lambda_{a n}=12, \quad \lambda_{\text {cath }}=15$ and the lowest performance at $\lambda_{a n}=4, \lambda_{\text {cath }}=5$ (extrapolated) is about 450 $\mathrm{mV}$, which affects the affects the performance of the MEA by $\sim 12 \%$.

The loss of performance for low stoichiometry ratios at high current densities might be associated with two reasons. The first is due to lower feed of reactants. The reactants are consumed along the channels hence causing a descending partial pressure in the gas channels. Additionally high mass transport of reactants form the through gas diffusion layer provokes reactants concentration gradient between the gas channel and the catalyst layer. This results in lowering of the overall

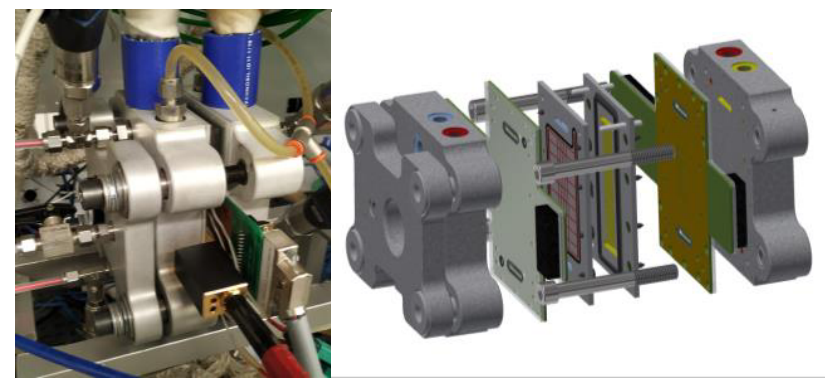

Fig. 4. Assembly of segmented current collector for the JRC ZEROVCELL and its installation on the testing station.

MEA performance. The second reason could be associated with less efficient removal of product liquid water at low stoichiometry ratios. The lower mass flow rate of reactant gasses results in lower pressure drop, which in turn has an effect on the efficiency of liquid water evacuation form the active area of the MEA. Nevertheless, considering wide range of considered reactant stoichiometry ratios (from $\lambda_{\text {cath }}=12$ down to $\lambda_{\text {cath }}=4$ ), the effect on the performance equal to $\sim 12 \%$ at the highest current densities only can be considered as relatively low.

The current density gradients across the MEA active area can be considered in two directions: along the gas channels (lines 1-4) and perpendicular to the parallel channels in the flow field, namely rows $a$ to $e$, Fig. 2a. The distribution on the current density along gas channels shows the MEA performance change due to changes in reactant gas composition and liquid water effects. On the other hand, the current density distribution in transverse direction is able to investigate the uniformity of MEA performance at comparable gas/liquid composition (among parallel channels) and identify effects of the active area sides. In order to further extend the range of investigated current densities, the evaluation of the current density distribution at various reactant stoichiometry ratios were done at fixed operating voltage of $0.3 \mathrm{~V}$, which corresponds to current density range of $3.7 \div 4.8 \mathrm{Acm}^{-2}$, depending on applied reactant stoichiometry ratio.

The plots of the current density distribution in both considered directions for a range of reactant stoichiometry ratios are presented in Fig. 6. It is clearly visible, see Fig. 6a, that distribution of current densities

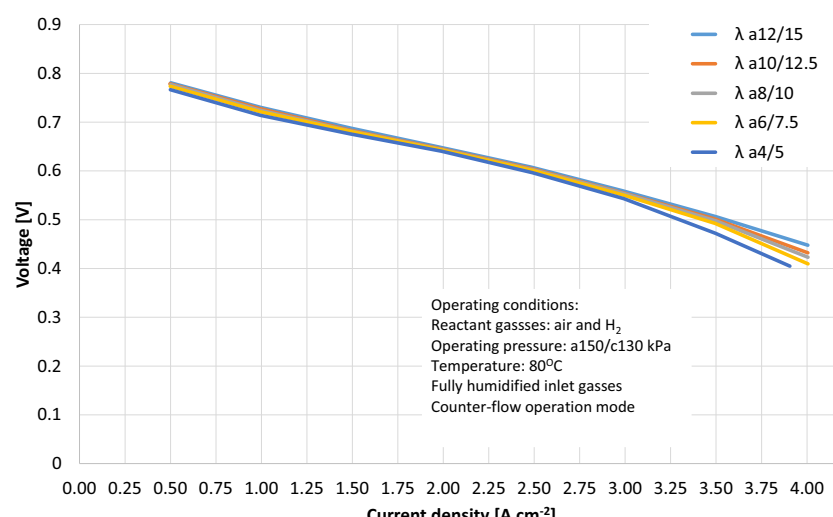

Fig. 5. Polarisation IV curves for a range of reactant stoichiometry ratios, from $\lambda_{a n}=4, \lambda_{\text {cath }}=5$ up to $\lambda_{a n}=12, \lambda_{\text {cath }}=15$. 
a)

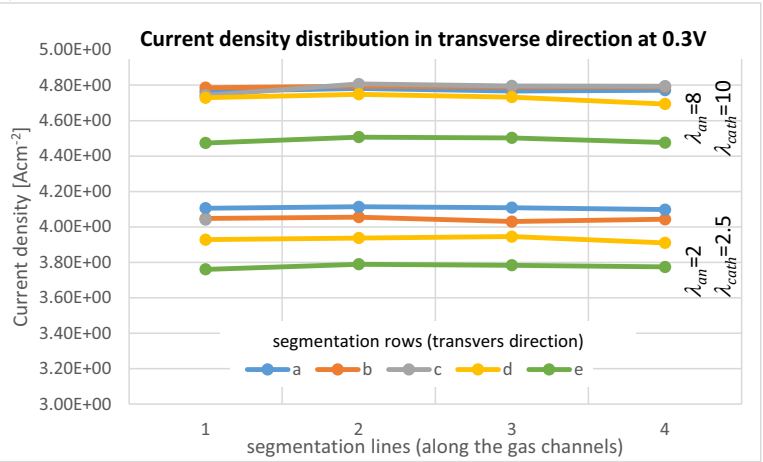

b)

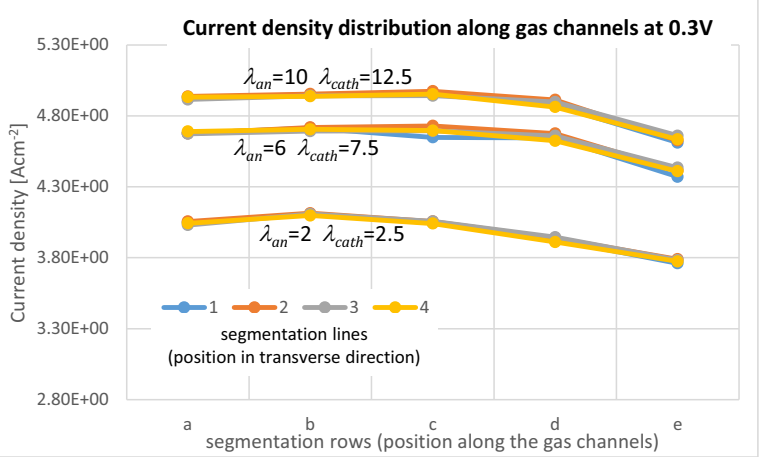

Fig. 6. Distribution of current density at steady operation at $0.3 \mathrm{~V}$ for selected stoichiometry ratios: (a) in transvers direction and (b) along the gas channels,

in the transverse direction is nearly uniform in consecutive positions along the channel. Spread of the corresponding values slightly exceeds measurement accuracy, namely $\pm 3 \mathrm{~mA}$. Therefore, it is shown that working conditions in all individual parallel channels are equal and there are no further effects of the sides of the active area.

The current density distribution for selected reactant stoichiometry ratios in longitudinal direction (lines 1-4) is presented in Fig. 6b. Since the current density distribution in transverse direction is rather uniform, the lines 1-4 at corresponding operating stoichiometry ratios are overlapping. It can be seen, Fig $6 \mathrm{~b}$, that in the first three rows the current density is rather uniform independently on the operating stoichiometry ratio. This would mean that reactant concentration in the gas channel is still high enough to efficiently feed the fuel cell reaction in the catalyst layer. However, in the following rows $d$ and $e$ the performance of the MEA slightly decreases, depending on the applied reactants stoichiometry ratio. Such performance loss can be associated with both, decrease of partial pressure of reactants due to their consumption and limited diffusion of reactants to the catalyst layer due to hindering effects of liquid water accumulated already in the gas channel from rows $a$ to $c$

However, comparison of current distribution curves is difficult due to different values of the total current produced by the MEA. In order to enable fair evaluation of the effects of the reactant stoichiometry ratios on current density distribution, its longitudinal gradient is being calculated and presented in Fig. 7. It can be seen that the current density gradients are rather independent of the operating stoichiometry ratio. Additionally, current

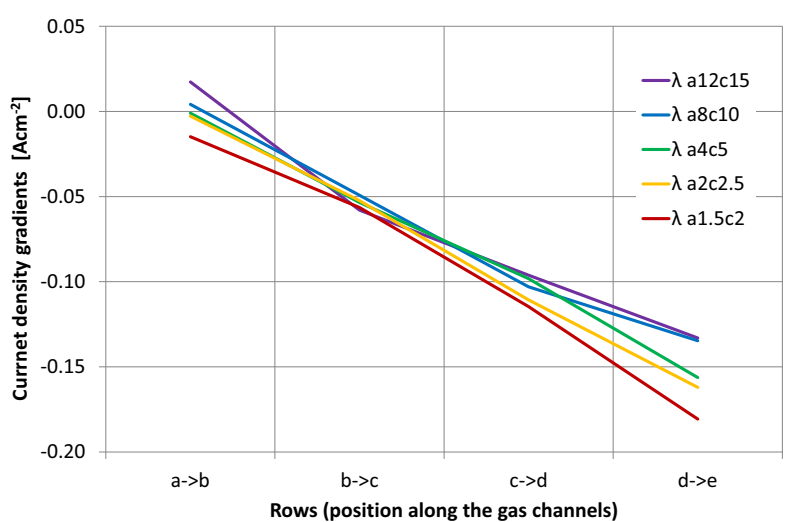

Fig. 7. Current density gradients along the gas channels at various reactant stoichiometry ratios.

density gradients show linear behaviour from the beginning of the active area (row $a$ ) down to the end of the active area (row $e$ ), what could suggest linear reasons of performance loss along the channels. Such linear reasons could be associated with linearly descending partial pressure of reactants, as opposite to negative effects of liquid water affecting diffusion of reactants, which are expected to be non-linear. However, in order to separate effects due to liquid water and reactants consumption a more detailed analysis is required using i.e. computer simulations.

\section{Conclusions}

The evaluation of current density distribution across the active area of the JRC ZEROVCELL single cell PEM fuel cell testing hardware was done using segmented current collector and semi-segmented bi-polar plate at a range of reactant stoichiometry ratios. The results show that the overall cell performance, although dependant on reactant stoichiometry ratios, varies $\sim 12 \%$ in the stoichiometry ratios range from $\lambda_{a n}=2, \lambda_{\text {cath }}=2.5$ up to $\lambda_{\text {an }}=12, \lambda_{\text {cath }}=15$. Current density distribution in the direction transverse to the gas channels is uniform, hence there are no effects of the active area sides, neither other effects affecting performance across the active area of the testing hardware. In the direction along the gas channels, gradients of current density show linear behaviour in the whole range of investigated stoichiometry ratios. That would suggest linear phenomena causing decrease of the cell performance along the channel such as decreasing reactants partial pressure and diffusion resistance, as opposite to liquid water effects, which are expected to be non-linear.

The design documentation of the JRC ZEROVCELL is publicly available at DOI: $10.17632 / \mathrm{c} 7 \mathrm{bffdv} 7 \mathrm{yb} .1$ under CERN Open-Hardware licence.

\section{References}

1. T. Bednarek. G. Tsotridis, J. Power Sources, 473, (2020)

2. T. Bednarek, DOI: $10.17632 / \mathrm{c} 7 \mathrm{bffdv} 7 \mathrm{yb} .1$

3. T. Bednarek. G. Tsotridis, Data Brief, 27, (2020) 